\title{
Fluorescence Emitted by Papanicolaou-Stained Urothelial Cells Improves Sensitivity of Urinary Conventional Cytology for Detection of Urothelial Tumors
}

\author{
Kinan Drak Alsibai ${ }^{\mathrm{a}, \mathrm{i}}$, Ghislaine Daste ${ }^{\mathrm{b}}$, i,, , Sophie Ferlicot ${ }^{\mathrm{c}}$, Monique Fabre ${ }^{\mathrm{d}}$, \\ Karine Steenkeste ${ }^{\mathrm{e}}$, Julia Salleron ${ }^{\mathrm{f}}$, Yacine Hammoudig, \\ Marie-Pierre Fontaine-Auparte, Pascal Eschwege ${ }^{\mathrm{h}}$
}

\begin{abstract}
Background: Urinary conventional cytology (UCCy) is easy to perform, but its low sensitivity, especially for low-grade urothelial neoplasms (LGUNs), limits its indications in the management of patients at risk of bladder cancer. The authors aim at obtaining a complementary test that would effectively increase the sensitivity of UCCy on voided urines by analyzing fluorescence of Papanicolaou-stained urothelial cells with no change of method in slide preparation.
\end{abstract}

Methods: In this retrospective study of 155 patients, 91 Papanicolaou-stained voided urines were considered satisfactory under fluorescence microscopy (FMi). The results of FMi were compared with UCCy (using transmission microscopy) and correlated to cystoscopy, histology and follow-up data.

Results: The results are given for all patients and for two groups of them according to the patients' main complaints (group 1: 33 patients

Manuscript submitted June 11, 2020, accepted August 12, 2020

Published online October 15, 2020

aService d'Anatomie Pathologique, Centre des Ressources Biologiques, Centre Hospitalier de Cayenne, 97306 Cayenne Cedex, France

${ }^{b}$ Centre de Pathologie Cellulaire et Moleculaire (CPCM-Labs), 31750 Escalquens, France

'Service d'Anatomo-Pathologie, Universite Paris-Sud, Hopital Bicetre, Assistance Publique-Hopitaux de Paris, 94276 Le Kremlin-Bicetre Cedex, France

dService d'Anatomie Pathologique, Universite Paris-Descartes, Hopital Universitaire Necker-Enfants Malades, Assistance Publique-Hopitaux de Paris, 75743 Paris Cedex, France

'Institut des Sciences Moleculaires d'Orsay (ISMO), CNRS, Universite et Paris-Saclay, 91405 Orsay Cedex, France

fService de Biostatistiques, Institut de Cancerologie de Lorraine, 54519 Vandoeuvre-les-Nancy Cedex, France

gService d'Urologie, Universite Paris-Sud, Hopital Bicetre, Assistance Publique-Hopitaux de Paris, 94276 Le Kremlin-Bicetre Cedex, France

${ }^{\mathrm{h}}$ Service d'Urologie, Universite de Lorraine, CNRS UMR 7039 CRAN, Centre Hospitalier Regional Universitaire, 54035 Nancy Cedex, France

iThese authors should be considered joint first authors.

${ }^{j}$ Corresponding Author: Ghislaine Daste, Centre de Pathologie Cellulaire et Moleculaire (CPCM-Labs), 61 Avenue de Toulouse, 31750 Escalquens, France. Email: gdacp31@gmail.com

doi: https://doi.org/10.14740/wjon1305 followed up for a previously treated bladder tumor; group 2: 58 patients with persistent urinary symptoms). Overall negative predictive value (NPV) and sensitivity of FMi were $100 \%$ vs. $73.7 \%$ and $64.3 \%$ respectively for UCCy $(\mathrm{P}=0.0001)$. Sensitivity of FMi for LGUN was unexpectedly high with a value of $100 \%$ vs. $46.2 \%$ for UCCy $(\mathrm{P}=0.0002)$. FMi was significantly superior to UCCy for detecting urothelial tumors in every group of patients and would allow a better characterization of atypical urothelial cells (AUCs) defined by the Paris System for Reporting Urine Cytology (TPS).

Conclusions: Because of its sensitivity and NPV of 100\%, FMi could complement UCCy to screen voided urines allowing a better detection of primary urothelial tumors or early recurrences of previously treated urothelial carcinoma. Moreover, this "dual screening" would allow completing efficiently cystoscopy to detect flat dysplasia, carcinoma in situ (CIS) and extra bladder carcinoma.

Keywords: Voided urines; Papanicolaou stain; Cytology; Fluorescence; Urothelial tumors

\section{Introduction}

Bladder urothelial carcinoma is the 10th most frequent cancer worldwide and about 543,000 new cases were diagnosed in 2018 [1]. The techniques used for the primary diagnosis and the follow-up of urothelial tumors are currently cystoscopy and urinary conventional cytology (UCCy). Cystoscopy has a good sensitivity for detection of urothelial tumors that varies between $69.2 \%$ and $97 \%$ depending on the type of cystoscope and the methodology (white light endoscopy and photodynamic diagnosis) $[2,3]$. But it is an operator-dependent, invasive and expensive medical tool which often fails to detect flat tumors such as carcinoma in situ (CIS) and is not suited for the detection of upper urinary tract tumors. UCCy is an easy and reliable technique for cytopathologists with high specificity but low overall sensitivity. Several studies reported its excellent sensitivity $(80-90 \%)$ in the detection of high-grade urothelial carcinoma (HGUC) and its dramatic decrease to $12-17 \%$ for grade 1 (G1) and to $26-61 \%$ for grade $2(\mathrm{G} 2)$ carcinomas $[4,5]$. At that time, it was difficult to accurately assess UCCy's performance in G1 
and G2 carcinomas, due to low inter-observer reproducibility in cytology and histology $[6,7]$. These findings partly explain evolution in successive editions of the World Health Organization (WHO) classification over the last 30 years. Consequently, since 2004, most of G1 and half or two-thirds of G2 carcinomas have been considered as low-grade urothelial carcinomas (LGUCs).

Another problem is the identification of atypical urothelial cells (AUCs) in urine. This category remained undefined during many years and its cytodiagnosis was very subjective. As a result, a diagnosis of AUC included specimens with little chance of a significant injury as well as specimens with higher risk of malignancy. According to a study carried out in 2013 on 16,299 cytology specimens, an $8 \%$ atypia rate was reported with only $21 \%$ of these progressing to a malignant diagnosis [8]. Others authors tried to better define AUC by analogy with the Bethesda System for cervical smears and separated AUCUS (atypical urothelial cells of undetermined significance) and AUC-H (atypical urothelial cells cannot exclude high grade) specially Piaton et al [9]. In 2016, the Paris System (TPS) clarified the morphological criteria necessary to include abnormal cells in the "AUC" category [10]. The comparative studies before and after TPS show that its stringent cellular criteria significantly decrease AUC category and increase the sensitivity of UCCy in diagnosing HGUC but do not improve diagnosis of low-grade urothelial neoplasms (LGUNs) [11-15].

In order to address the shortfalls of UCCy, different urinary biomarkers have been introduced as a complement or substitution for UCCy $[16,17]$. These tests are based on the immunodetection of several antigens or on molecular biology techniques. ImmunoCyt/uCyt $\mathrm{tM}^{\mathrm{TM}}$ is an immunofluorescence assay and UroVysion ${ }^{\mathrm{TM}}$ FISH is a multitarget fluorescence in situ hybridization (FISH) that detects aneuploidy [18-20]. BTA TRAK $^{\circledR}$ and NMP $22^{\circledR}$ Test Kit quantify respectively the human complement factor H-related protein and the nuclear matrix protein 22 in urine $[21,22]$. BTA stat ${ }^{\circledR}$ and NMP $22^{\circledR}$ Bladder Chek are only qualitative tests $[23,24]$. These tests have a variable sensitivity and often a lower specificity than UCCy [25]. They require specialized technical scores and expensive equipment, specific skills of pathologists and preparation of additional samples. As a consequence, their high cost/benefit ratio limits their access to an entire population [26].

More recently molecular tests study mutations, methylation or expression of specific genes [27-29]. Specially Kavalieris et al [29] described the Cxbladder Monitor combining phenotypic (clinical and patient data) and genotypic (gene expression) urinary biomarkers. This test exhibited sensitivity and negative predictive value (NPV) of 93\%, outperforming other tests as NMP22 Blad$\operatorname{der}$ Chek $^{\circledR}$ and UroVysion ${ }^{\circledR}$ FISH. The authors concluded that the use of Cxbladder Monitor could reduce the number of cystoscopies for the evaluation of recurrent urothelial carcinoma and is able to accurately triage out patients who present hematuria [30].

Actually, although these tests including the Cxbladder Monitor are approved by Food and Drug Administration (FDA), they are not recommended in American Urological Association (AUA) or European Association of Urology (EAU) guidelines for detection or follow-up of bladder cancer [31, 32]. Only UroVysion ${ }^{\circledR}$ FISH can be used to assess response to intravesical BCG and UroVysion ${ }^{\circledR}$ FISH and ImmunoCyt ${ }^{\mathrm{TM}}$ to adjudicate on AUC in patients with non-muscle-invasive bladder cancer
(NMIBC) (expert opinion, AUA) [31].

Liquid-based cytology (LBC) procedures have allowed improving the slides cellularity and decreasing the rate of unsatisfactory slides, but did not significantly improve the sensitivity of cytodiagnosis for LGUN compared with cytocentrifugation methods [33, 34].

Hence, despite its poor performance for LGUN and its difficulty to characterize atypical cells, UCCy is still the preferred diagnostic tool. The search for alternative or complementary methods, offering both performance and simplicity, is therefore critical for the improvement of the diagnosis of LGUN.

Some studies have reported fluorescence microscopy observation of dysplastic or tumoral cells. Millot et al [35] reported a particular auto-fluorescence emission from cells of high- and low-grade intra-epithelial lesions on unstained cervical smears. Another study reported an induced fluorescence on urothelial cells of HGUC (15/16 cases), LGUN (26/31 cases) and CIS (9/11 cases) from bladder washing after endoscopy with 5-aminolevulanic acid [36]. In our first publication [37], we studied and monitored the fluorescence properties of classical Papanicolaoustained urothelial cells from urines or cell lines to investigate metabolic changes in normal and tumoral cells. We observed different fluorescence patterns on urothelial cells in various clinical conditions. An intense peri-membrane fluorescence was always observed on urothelial carcinoma cells but also on some reactive or regenerative urothelial cells occurring in inflammation or lithiasis and contrasting by intra-cellular localization and low intensity fluorescence of normal, quiescent urothelial cells. These different fluorescence patterns could be used for detecting urothelial carcinoma in addition to UCCy. This method does not require supplementary urinary samples nor changes to the Papanicolaou staining protocol, unlike other methods using fluorescence as a photodynamic diagnosis [36, 38].

In this current work, we presented a comparative study of performances of FMi and UCCy on voided urines of patients before cystoscopy and evaluated the role of both combined methods in diagnosis of urothelial tumors.

\section{Materials and Methods}

\section{Patients' cohort}

We report a monocentric retrospective study of 155 archived consecutive cases in the cystoscopy's register of Urology Department at Bicetre Hospital from January 2011 to January 2012. For all patients, a voided urine specimen has been collected before cystoscopy. After exclusion of 26 cases with incomplete clinical report, 17 others with final diagnosis of non-urinary tract cancer (kidney, prostate, etc.) and 11 unsatisfactory urinary slides, 101 patients (19 women and 82 men) were selected (Fig. 1). The mean age was 71.2 years (range $19-95$ ).

\section{Control cases}

In order to complete the validation of FMi on normal urothelial cells, we collected voided urines from 22 healthy volunteers, 


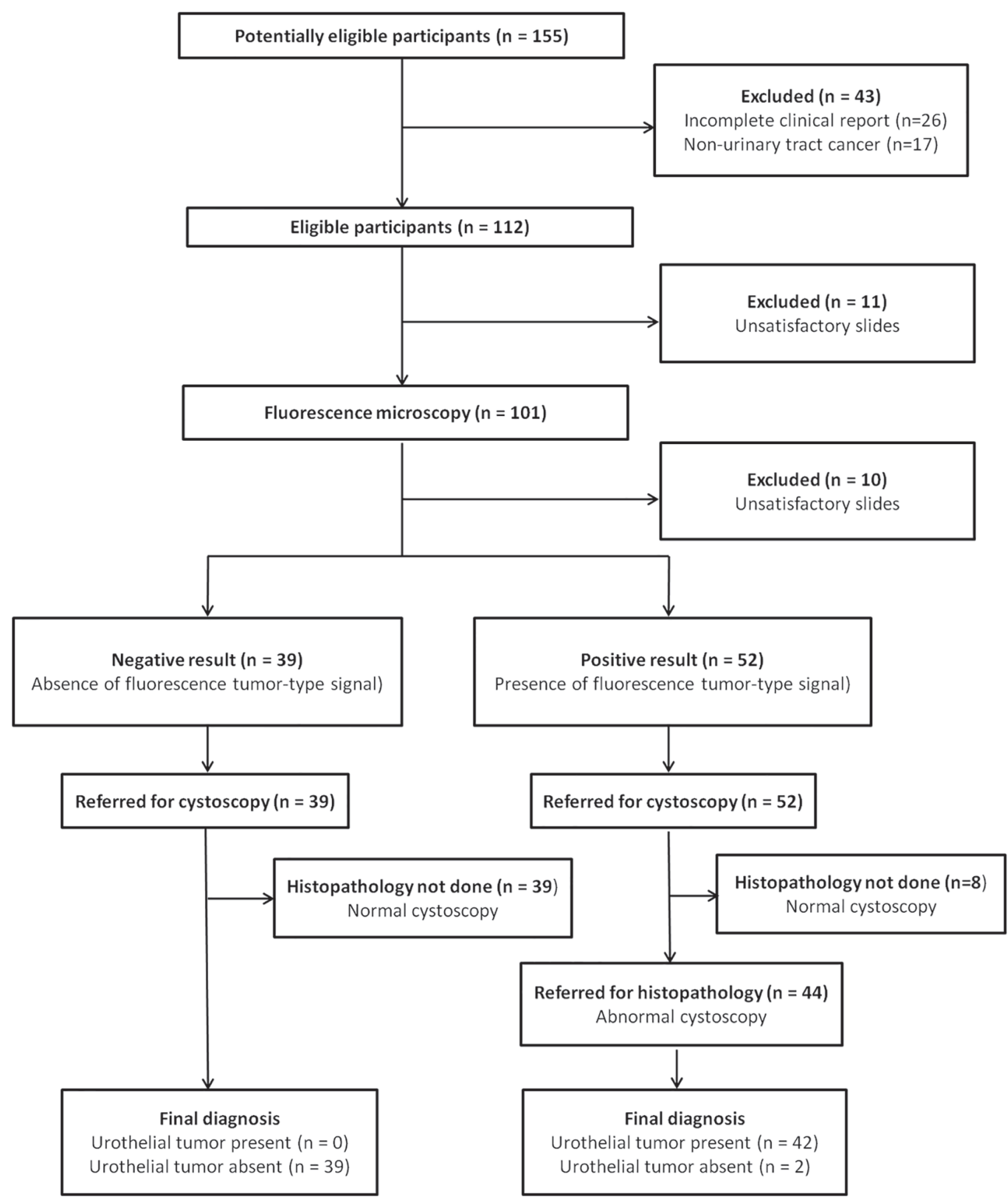

Figure 1. Diagram of STARD.

18 men and four women between 18 and 63 years, exempt from any urinary tract pathology and without risk factors.

\section{Urine cytology slides preparation}

Before a standard white light cystoscopy, $30 \mathrm{~mL}$ of voided urines were collected in a sterile recipient that contained $10 \mathrm{~mL}$ of $50 \%$ ethyl alcohol (EtOH), and transferred to the pathology depart- ment.

After centrifugation of the entire sample at 3,000 rpm during $5 \mathrm{~min}$, the supernatant was discarded and the pellet was resuspended in $1 \mathrm{~mL}$ (when pellet was small) to $2 \mathrm{~mL}$ of $0.9 \%$ $\mathrm{NaCl}$. Successively, $400 \mu \mathrm{L}$ of the resuspended pellet was transferred in a settling chamber of Shandon Cytospin 2 (Thermo-Fisher, Waltham, USA) for $10 \mathrm{~min}$ at $800 \mathrm{rpm}$ followed by slides spray fixation then Papanicolaou staining.

The manual procedure of Papanicolaou staining was as fol- 
lows. The slides were immersed in $95 \% \mathrm{EtOH}$ for $10 \mathrm{~min}$ and successively rehydrated in 50\% EtOH (1 min) and in running water (1 min). The slides were then stained with Harris hematoxylin (ref 361075 RAL Diagnostics, Martillac, France) for $3 \mathrm{~min}$ and transferred successively, in running water (1 min), briefly in $0.2 \% \mathrm{HCl}$ diluted in ethanol $(5 \mathrm{~s})$, in running water $(1$ $\min )$, in $0.2 \% \mathrm{NH}_{4} \mathrm{OH}(30 \mathrm{~s})$, in running water $(1 \mathrm{~min})$, in $95 \%$ $\mathrm{EtOH}(1 \mathrm{~min})$ and in $100 \% \mathrm{EtOH}(1 \mathrm{~min})$. Next the slides were stained with Orange G6 (ref 361630 RAL Diagnostics, Martillac, France) for 2 min then immersed in two 100\% EtOH baths for $1 \mathrm{~min}$, each. This step was followed by a bath in EA 50 (ref 367600 RAL Diagnostics, Martillac, France) for $3 \mathrm{~min}$ and then in two $100 \% \mathrm{EtOH}$ baths for 1 min each.

The slides were then covered with the film Tissue-Tek ${ }^{\circledR}$ Coverslipping (Sakura, Finetek, Europe) after two xylene baths for $2 \mathrm{~min}$.

The same technical procedure was applied to voided urines collected from one natural micturition in volunteers.

\section{Tissue specimen treatment}

Biopsy or transurethral resection for bladder tumor under white light or cystectomy was performed by the same academic urologic surgeon.

Conventional histopathological techniques were applied (formol fixation, paraffin inclusion, hematoxylin-eosin coloration).

\section{Microscopic observation}

A conventional microscope (Olympus BX 51, Tokyo, Japan) equipped with a continuous mercury-arc lamp (X-cite 120Q, EXFO) and a CDD camera was used for observation with white and fluorescent light. Excitation and emission fluorescence ranges were selected through bandpass filters.

\section{Fluorescent mode}

In our previous study [37], we have tried to explain the different fluorescence patterns observed on quiescent and reactive/regenerative or tumoral urothelial cells after Papanicolaou staining. Analysis of fluorescence emitted by the different dyes of Papanicolaou staining and by urothelial cells before and after Papanicolaou staining, allowed concluding that the fluorescence was not caused by cell endo-fluorophores but essentially by accumulation of EA50 in some cell constituents. This 2007 study allowed us to define an excitation wavelength between 410 and $510 \mathrm{~nm}$ and an emitted fluorescence collected at wavelengths between 520 and $580 \mathrm{~nm}$. Low intensity and intra-cellular localization characterized fluorescent signal of urothelial normal cells (FNs) while an intense and peri-membrane fluorescence called fluorescent tumor-type signal (FTs) was emitted by urothelial tumoral or active/regenerative cells (after erosion caused by inflammation or lithiasis for example). Moreover, a fluorescence reactivity similar to the FTs was emitted by polynuclear leukocytes and was generally easy to recognize because of their nuclear forms.
Fluorescence observation was performed on the Papanicolaou-stained slides at $\times 20$ objective by a pathologist trained in fluorescence microscopy, unaware of patient status. This first FMi screening allowed classifying slides as not interpretable or positive FMi (with FTs) or negative FMi (without FTs). Next, by rapidly switching from fluorescence to transmission microscopic mode, this pathologist verified that fluorescence was effectively emitted by urothelial cells.

The consultation of clinical patients' reports allowed correlating results of FMi with 2011 - 2012 cytological/histological findings.

\section{Transmission mode}

Next, a pathologist specialized in Urinary Cytopathology and Histopathology, blinded to transmission and fluorescent microscopy results, reviewed each urinary slides according to 2016 TPS and all cases of surgical pathology according to the 2016 WHO tumor classification.

\section{Statistical method}

After inclusion in patient database and before statistical analyses, all patients' information was de-identified and not made available to researchers accessing this database. Because of this, our study was exempt from Institutional Review Board (IRB) approval. All patients have been managed in the Bicetre University Hospital according to the standards of good clinical practice and ethical approval.

The calculation of UCCy and FMi performances used the results of cystoscopy completed by histological analysis. The data were analyzed with the R software version 3.4.3. The McNemar test for paired study design was used to determine the difference in sensitivity and specificity between FMi and UCCy. In case of small number of discordant pairs $(\leq 10)$, an exact binomial test was performed. For NPV and positive predictive value (PPV), we used the method proposed by Moskowitz and Pepe [39].

\section{Results}

For a better understanding of the presentation of the FMi results in tables, cases with FTs were considered FMi positive and cases without FTs were considered FMi negative.

\section{Volunteers}

In the 22 volunteers, all slides were interpretable and negative for UCCy and FMi. So, in urines of healthy person (absence of inflammation, lithiasis, tumor), no cell was FMi positive.

\section{Patients' cohort (101 cases)}

Among the 101 included cases, FMi was not interpretable on 
Table 1. Results of Transmission and Fluorescence Microscopy in Groups 1 and $2(\mathrm{~N}=91)$

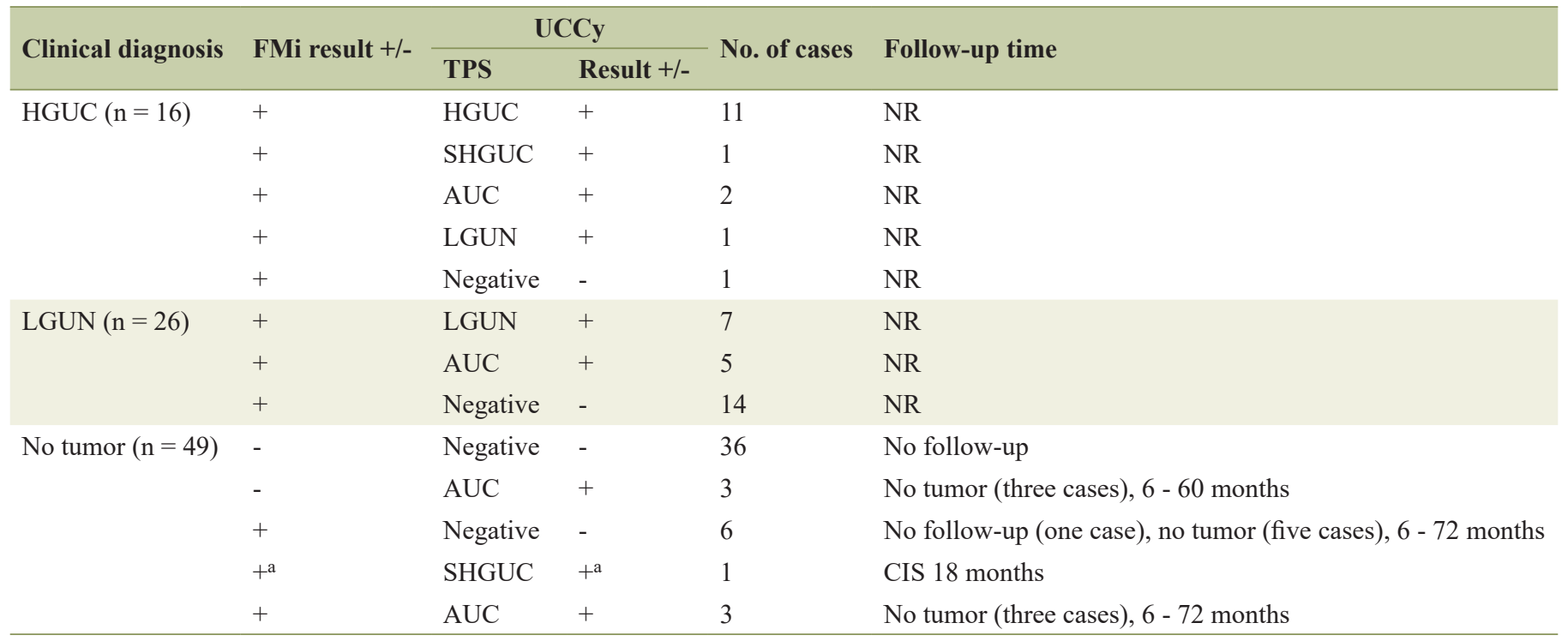

\begin{tabular}{llllll}
\hline Statistics & FMi & UCCy & P*(FMi vs. UCCy $)$ & FMi + UCCy & P* (FMi + UCCy vs. UCCy) \\
\hline $\begin{array}{c}\text { HGUC + LGUN } \\
\text { Sensitivity, \% }\end{array}$ & 100 & 64.3 & $0.0001 *$ & 100 & $0.0001 *$ \\
Specificity, \% & 79.6 & 85.7 & 0.3173 & 73.47 & $0.0143 *$ \\
PPV, \% & 80.8 & 79.4 & 0.8238 & 100 & 0.5051 \\
$\quad$ NPV, \% & 100 & 73.7 & $0.0001 *$ & & $0.0001 *$ \\
HGUC & & & & & \\
$\quad$ Sensitivity, \% & 100 & 93.7 & 0.3173 & & \\
LGUC & & & & & \\
$\quad$ Sensitivity, \% & 100 & 46.2 & $0.0002 *$ &
\end{tabular}

${ }^{a} \mathrm{CIS}$ diagnosed after 18 months. *Significant threshold: P < 0.05. AUC: atypical urothelial cell; CIS: carcinoma in situ; FMi: fluorescence microscopy; HGUC: high-grade urothelial carcinoma; LGUN: low-grade urothelial neoplasia; NPV: negative predictive value; PPV: positive predictive value; SHGUC: suspicious for high-grade urothelial carcinoma; TPS: The Paris System; UCCy: urinary conventional cytology.

10 slides because of a diffuse or a too low fluorescence probably related to impaired staining. On slides considered satisfactory under FMi, the number of abnormal urothelial cells with FTs varied between 3 and more than 100 per slide in our series.

Statistical analyses were applied on total patients cohort $(n=91)$ and on two groups of them separated according to the patient' chief complaint: group $1(\mathrm{n}=33)$ included patients followed up for a previous bladder tumor, group $2(\mathrm{n}=58)$ included patients with persistent urinary symptoms.

\section{All patients}

\section{Patients with urothelial tumor}

All 42 urothelial tumors (16 HGUCs and 26 LGUCs) were positive for FMi (sensitivity of 100\%), whereas overall sensitivity of UCCy was $64.3 \%(\mathrm{P}=0.0001)$. FMi was significantly superior to UCCy for detecting urothelial tumors with a sensitivity of $100 \%$ vs. $93.7 \%$ for HGUC (P=0.0001) and vs. $46.2 \%$ for LGUC $(\mathrm{P}=0.0002)$. The combination of the two tests together increased sensitivity and NPV to $100 \%$ (vs. $64.3 \%$ and $73.7 \%$, respectively, for UCCy alone, $\mathrm{P}<0.05$ ) and decreased specificity to $73.47 \%$ (vs. $85.7 \%$ for UCCy alone, $\mathrm{P}<0.05$ ).

An urothelial tumor was found in $70 \%$ of AUCs (7/10) associated with positive FMi. LGUC was found in about 2/3 of negative cytology cases associated with positive FMi (Table 1).

\section{Patients without urothelial tumor}

Of the 49 cases without a proven contemporary tumor, 36 cases were negative for UCCy and FMi. No tumor was found during the follow-up of all AUCs (six cases: three with positive FMi and three with negative FMi) and of all negative cytology cases $(n=6)$ with positive FMi. A CIS was diagnosed after 18 months in an SHGUC with positive FMi.

No tumor was detected during the contemporary examina- 


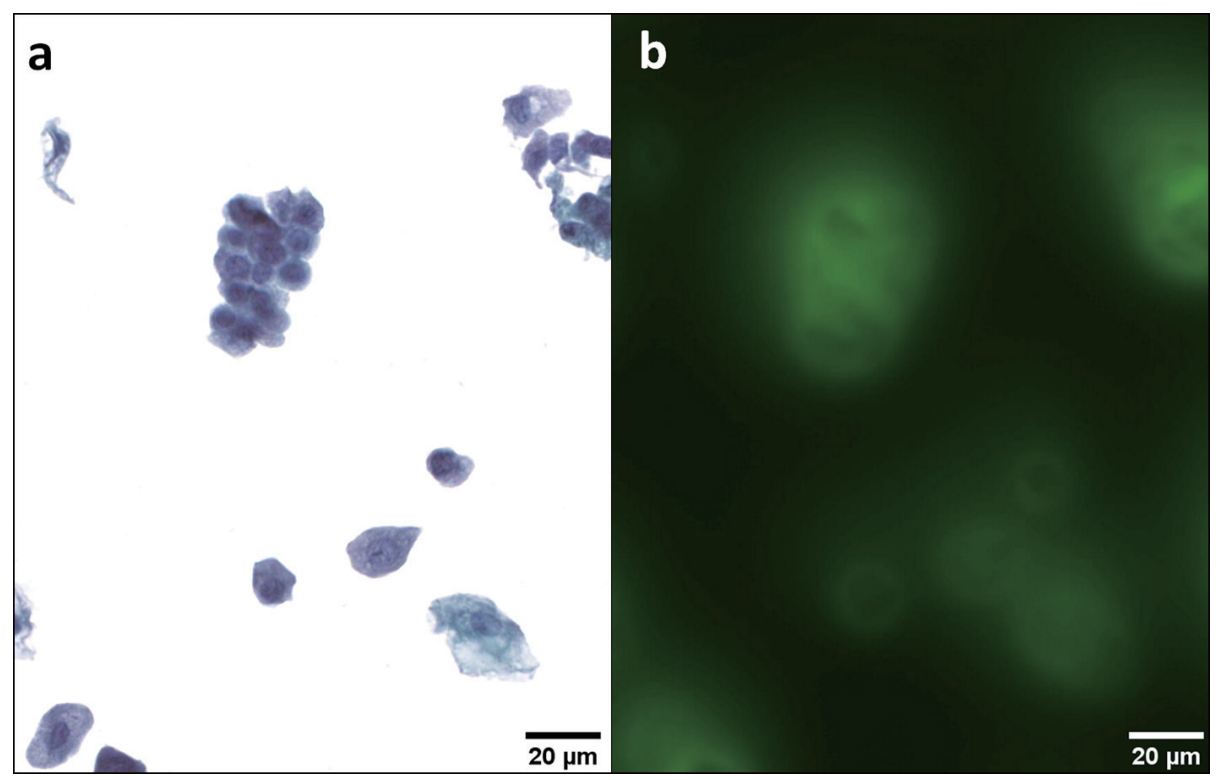

Figure 2. Normal urothelial cells. Intermediate and deep urothelial cells showing a nucleus with homogeneous chromatin, regular borders and a nuclear/cytoplasmic ratio $(\mathrm{N} / \mathrm{C})<0.5(\mathrm{a})$. These cells emit a homogeneous and low intracellular fluorescence signal (b). Note the same fluorescence pattern of the malpighian cell. Transmission (a) and fluorescence microscopic (b) observations of Papanicolaou-stained urothelial cells.

tion or during the follow-up in AUC associated with negative FMi.

In less than $20 \%$ of cases, FTs was not related to tumor but might be explained by modifications of cell morphology and/or cell physiology induced by different urinary disruptions (Table 1).

Transmission and fluorescence microscopic observations are illustrated in Figures 2-5.

\section{Group 1}

\section{Patients with urothelial tumor}

Among 33 patients monitored for previously treated urothelial tumor, 22 had a positive cystoscopy followed by surgery. The histopathological analysis found 17 LGUCs and five HGUCs.

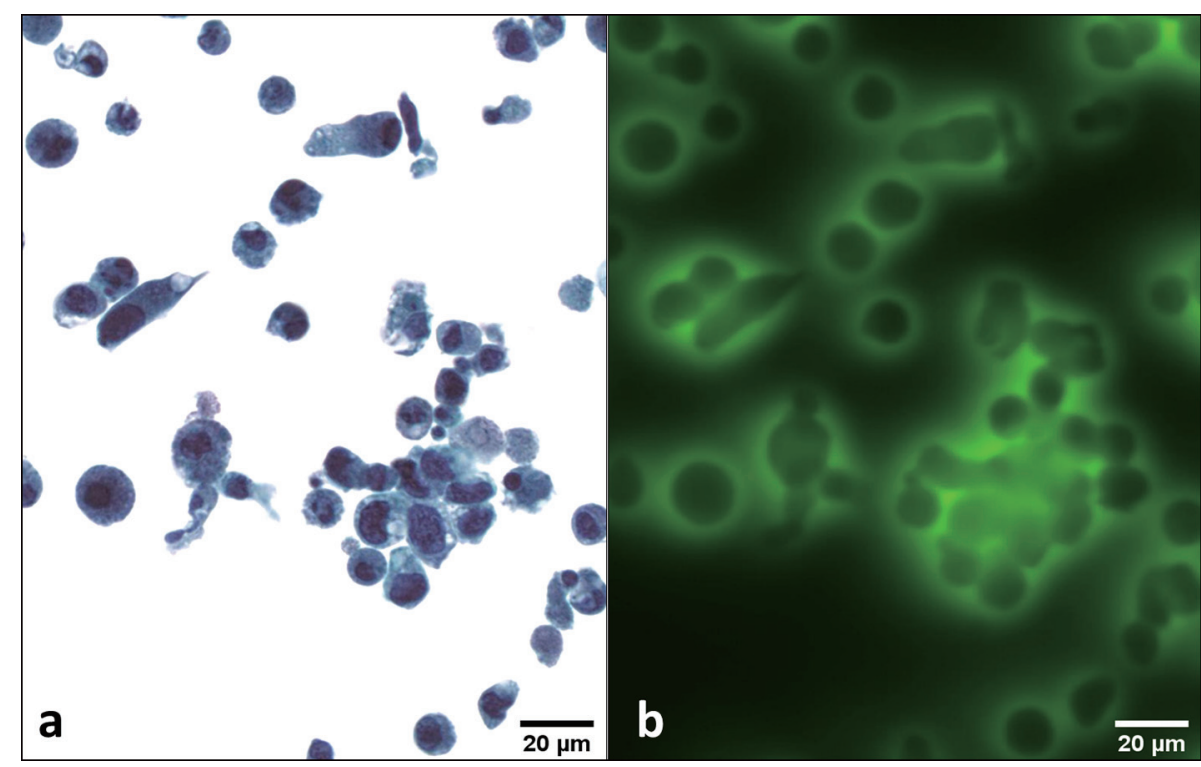

Figure 3. HGUC. Several urothelial deep cells are characterized by a high nuclear/cytoplasmic (N/C) ratio $>0.7$, coarse chromatin, hyperchromasia and irregular nuclear membrane; other cells show degenerative alterations (a). A strong and peri-membrane fluorescence signal is emitted by all these cells (b). Transmission (a) and fluorescence microscopic (b) observations of Papanicolaou-stained urothelial cells. HGUC: high-grade urothelial carcinoma. 


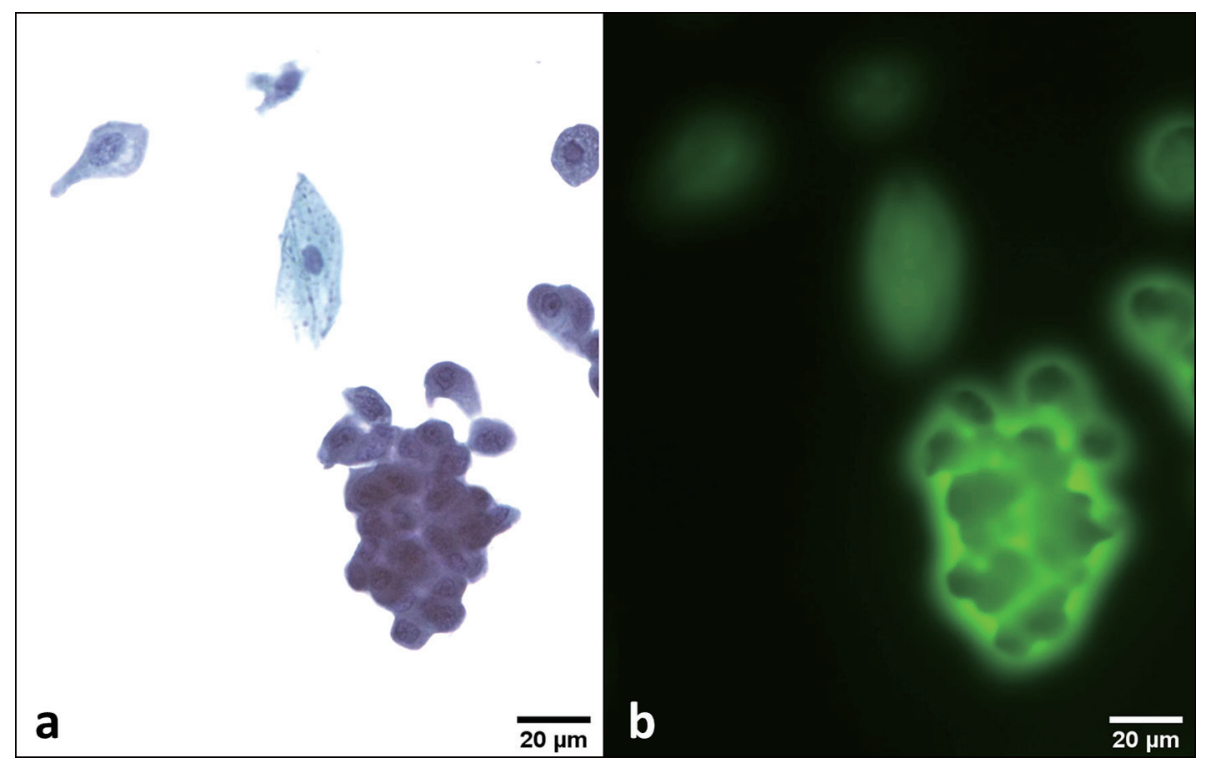

Figure 4. LGUN. A cluster of urothelial deep cells showing a slightly increased nuclear/cytoplasmic (N/C) ratio, sometimes eccentric and grooved nuclei (a). These cells emit a strong and peri membrane fluorescence signal (b). Transmission (a) and fluorescence microscopic (b) observations of Papanicolaou-stained urothelial cells. LGUN: low-grade urothelial neoplasia.

UCCy was negative in nine LGUCs and in one HGUC. AUCs were detected in three LGUCs and in one HGUC. UCCy was positive for HGUC in two HGUCs histologically confirmed. A cytology suggestive of LGUN was correlated with five LGUCs and one HGUC. FMi showed a FTs in nine LGUCs labeled negative with UCCy.

All 22 urothelial tumors were positive in FMi (sensitivity of $100 \%)$. Sensitivity of UCCy decreased significantly to $54.5 \%(\mathrm{P}=0.0016)$ (Table 2$)$.

\section{Patients without urothelial tumor}

Of 11 patients without a contemporary tumor, three had a positive FMi. Two of them were treated with Bacillus CalmetteGuerin (BCG) immunotherapy: in one case, the cytodiagnosis was AUC, the cystoscopy showed an inflammatory bladder and no tumoral recurrence was observed (followed by 3 years). On the contrary, in the second case labeled SHGUC with UCCy and contemporary negative biopsy, CIS was diagnosed 18

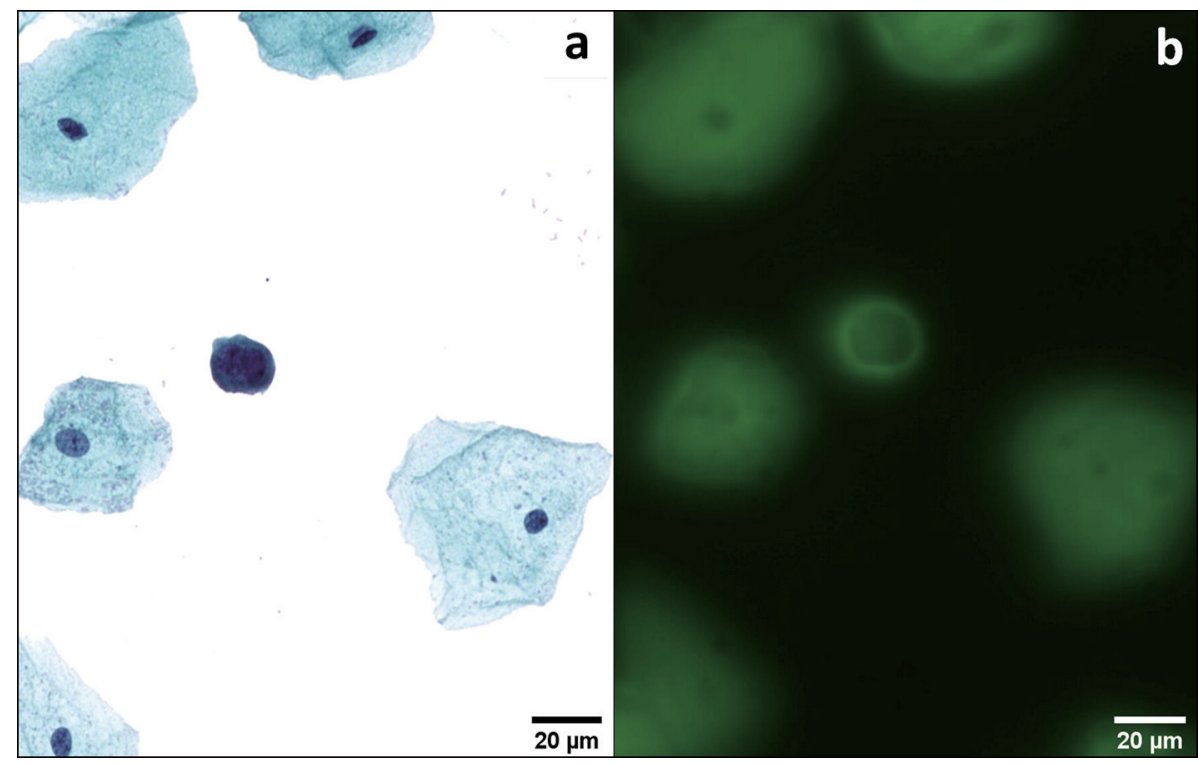

Figure 5. AUC. An atypical deep urothelial cell showing an increased nuclear/cytoplasmic (N/C) ratio $>0.5$ and a hyperchromatic nucleus (a). This cell emits a strong and peri membrane fluorescence signal (b). This patient had a positive cystoscopy and a final diagnosis of HGUC. Transmission (a) and fluorescence microscopic (b) observations of Papanicolaou-stained urothelial cells. AUC: atypical urothelial cell; HGUC: high-grade urothelial carcinoma. 
Table 2. Results of Transmission and Fluorescence Microscopy in Group 1 (33 Patients Followed Up for Previous Urothelial Neoplasms)

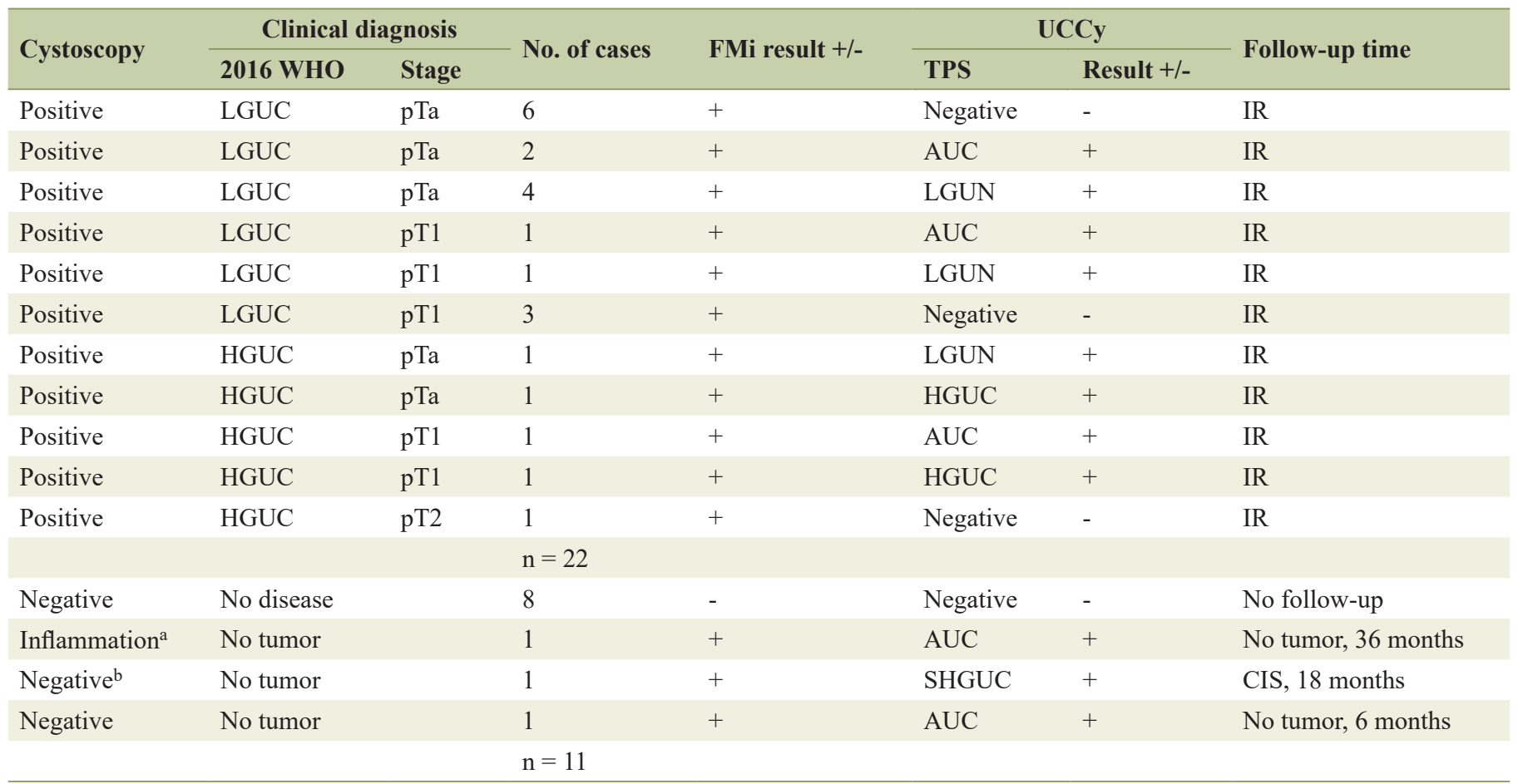

\begin{tabular}{llll}
\hline Statistics & FMi & UCCy & P* \\
\hline Sensitivity, \% & 100 & 54.5 & $0.0016^{*}$ \\
Specificity, \% & 72.7 & 72.7 & NA \\
PPV, \% & 88 & 80 & $0.0021^{*}$ \\
NPV, \% & 100 & 44.4 & 0.1289 \\
\hline
\end{tabular}

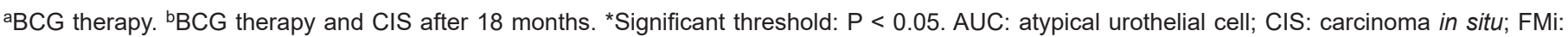
fluorescence microscopy; HGUC: high-grade urothelial carcinoma; IR: irrelevant; LGUC: low-grade urothelial carcinoma; LGUN: low-grade urothelial neoplasia; NPV: negative predictive value; PPV: positive predictive value; SHGUC: suspicious for high-grade urothelial carcinoma; TPS: The Paris System; UCCy: urinary conventional cytology; WHO: World Health Organization.

months later. So, UCCy and FMi probably detected abnormal cells long time before the diagnosis of CIS. For the last patient who had a partial cystectomy for pT2, we detected AUC with FTs in urines but no tumor was observed after a short 6-month follow-up (Table 2).

\section{Group 2}

\section{Patients with urothelial tumor}

Among 58 patients with persistent and unsolved urinary symptoms, 19 bladder and one ureteral urothelial tumors (nine LGUCs and 11 HGUCs) were confirmed by histology after surgery. UCCy did not detect five LGUCs. AUC was identified in two LGUCs and in one HGUC. SHGUC was consistent with one HGUC. UCCy was positive for HGUC in nine histologically confirmed HGUCs.
All 20 tumors showed FTs (sensitivity and NPV of 100\%). Sensitivity decreased significantly with UCCy to $75 \%(\mathrm{P}=$ 0.0253) (Table 3).

\section{Patients without urothelial tumor}

No tumor was diagnosed by cystoscopy and/or follow-up in 38 patients.

But in this second group, the false positive cases were more numerous: six cases with negative cytology exhibited FTs, three AUCs were negative FMi and one AUC was positive FMi. These 10 cases were related to lithiasis, bladder and prostate inflammation/infection, or benign prostate hyperplasia (BPH). None urothelial tumor was observed initially and during follow-up of 6 to 72 months for nine patients whereas the follow-up of one patient was missing.

Thus, PPV of FMi decreased to $74.1 \%$ in this second group in relation to the frequency of inflammations and lith- 
Table 3. Results of Transmission and Fluorescence Microscopy in Group 2 (58 Patients With Persistent Urinary Symptoms)

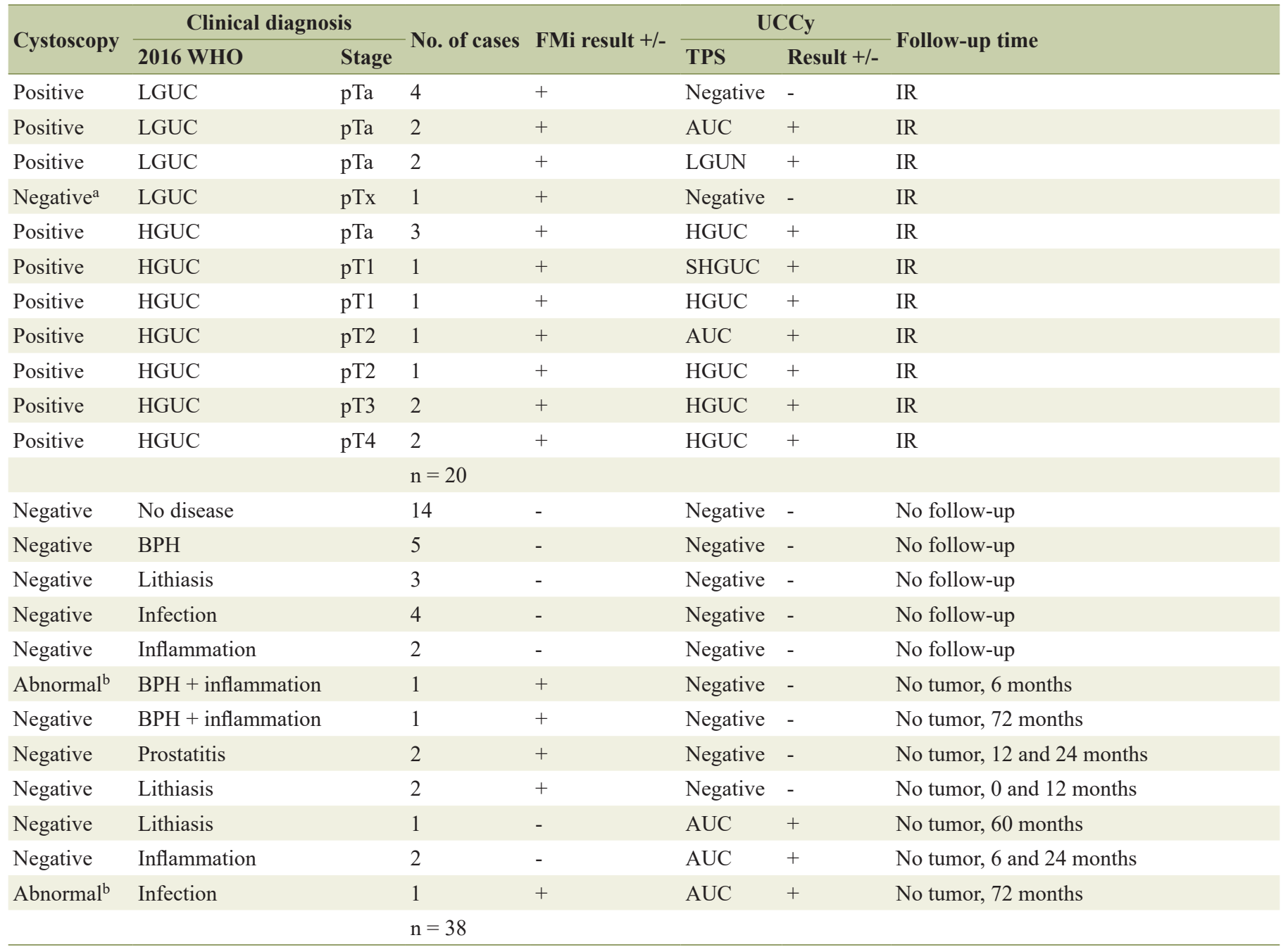

\begin{tabular}{llll}
\hline Statistics & FMi & UCCy & P* \\
\hline Sensitivity, \% & 100 & 75 & $0.0253^{*}$ \\
Specificity, \% & 81.6 & 89.5 & 0.3173 \\
PPV, \% & 74.1 & 78.9 & 0.6314 \\
NPV, \% & 100 & 87.2 & $0.0255^{*}$ \\
\hline
\end{tabular}

a. Ureteral urothelial tumor. ${ }^{b}$ Diagnosis confirmed by bladder biopsy. ${ }^{*}$ Significant threshold: $\mathrm{P}<0.05$. AUC: atypical urothelial cell; BPH: benign prostatic hyperplasia; FMi: fluorescence microscopy; HGUC: high-grade urothelial carcinoma; IR: irrelevant; LGUC: low-grade urothelial carcinoma; LGUN: low-grade urothelial neoplasia; NPV: negative predictive value; PPV: positive predictive value; SHGUC: suspicious for high-grade urothelial carcinoma; UCCy: urinary conventional cytology; WHO: World Health Organization.

iasis (Table 3 ).

\section{Discussion}

The cytopathologist has a pivotal role to play in the diagnosis of initial or recurrent urothelial tumors. Whereas UCCy can often detect HGUC, its sensitivity for diagnosing LGUN is low and requires considerable expertise.
This relative poor sensitivity of UCCy for LGUN may be explained by the fact that these tumors are less likely to shed cells in the urine as the tumor cells are more cohesive than in cases of HGUC. When LGUC or papillary urothelial neoplasm of low malignancy potential (PUNLMP) does exfoliate in urines, we observe clusters rather than single cells with mild or inconspicuous cellular atypia. The interpretation of these subtle changes is difficult and often unfocused because their cytological appearance is closer to that of normal urothelial 
cells and explains inter-observers variations [6, 7]. FMi as in others studies $[18,36]$ seems to confirm that the presence of low-grade tumoral cells in urines is relatively frequent but often undetectable with UCCy. Thus, Mian et al [8] reported a significant increase in sensitivity of about $80 \%$ for the detection of LGUC with the uCyt+/Immunocyt test used alone or coupled to UCCy.

Several authors have tried to better define AUCs [9, 4042]. Recently, TPS has attempted to outline some of the most common patterns that may be encountered in atypical urine specimens and their associated risk of an underlying urothelial neoplasm [10]. The criteria outlined by TPS facilitated the standardization of urine cytology report, significantly increased the sensitivity of diagnosing HGUC and refined the category "AUC" but did not improve diagnosis of LGUN [13]. So for some authors, there was histological evidence of urothelial carcinoma in $70 \%$ of AUCs after the TPS [12]. This finding was not confirmed by another study [43].

In addition, the UCCy interpretation was challenged by the fact that lithiasis, infections, urinary tract instrumentation and BCG immunotherapy may cause changes in the urine cytology features, and in turn lead to an erroneous positive cyto-diagnosis [44]. A large part of these reactive atypia would not be recruited into the AUC category after re-evaluating the same samples according to the TPS criteria [13].

Several biomarkers have emerged to help the early detection of bladder carcinoma. Although some are more sensitive than UCCy, they are usually less specific and some of them have a low sensitivity for LGUN. So few have made the successful transition into the clinic $[16,45]$. For the diagnostic of de novo tumors and monitoring of recurrent bladder cancer, cystoscopy stands as the best reference method. UCCy must be associated with cystoscopy during the surveillance of intermediate and high-risk patients and can be used as an adjunct to cystoscopy to detect de novo bladder tumor in patients with hematuria or other suggestive symptoms $[31,32]$.

Nevertheless, a significant improvement of cytological detection for LGUN would allow better managing the majority of patients. The annual incidence rate of bladder cancers varies from 2.3 to 32 per 100,000 depending on the countries, of which $70 \%$ are NMIBCs. These NMIBCs have a very high recurrence rate (up to $70 \%$ within 2 years of primary treatment) and $10-30 \%$ will develop to invasive disease after treatment. The most of them are low-grade Ta/T1 primary tumors $[1,30$, 46]. Among the LGUCs, $40 \%$ will recur and $15 \%$ progress in high-grade carcinoma. Among the HGUCs, 70\% will recur and $30 \%$ will progress to muscle-invasive carcinoma [46].

The results obtained with FMi seemed to improve UCCy sensitivity for high- and low-grade urothelial tumors.

1) Improvement of LGUN detection. FMi, with a sensitivity and NPV of $100 \%$ filled UCCy insufficiency. This gain is particularly important for LGUN detection, even in the absence of obvious changes in cellular morphology. In our series, the association of a FTs and a negative cytology was correlated with 15 LGUCs and one HGUC in histology.

2) AUC characterization. FMi appears to be a good tool for confirming the tumor nature of uncertain diagnosis as AUC. When AUC was associated with FTs, these atypia were related to an urothelial tumor in about $70 \%$ of cases. The phe- notype associating AUCs and NFs was never associated with a histologically confirmed tumor in our series.

Almost $50 \%$ of AUCs and less than $20 \%$ of FTs were not related to tumor but might be explained by modifications of cell morphology and/or cell physiology induced by different urinary disruptions.

3) Methodology. In contrast to the existing biomarkers that are more time-consuming, more expensive and require the preparation of additional samples, fluorescence localization after Papanicolaou staining is quickly performed on the same slides than UCCy.

For all these reasons, we think that FMi coupled with UCCy is able to accurately triage out patients who have a low probability of UC with a high overall test-negative rate, high level of sensitivity and high NPV.

These results will be confirmed in a larger number of patients with tumoral or benign urologic conditions, which is currently the object of another ongoing clinical study.

In addition, an automated process for analyzing cells as described in the patent [47] will be completed by an algorithm built with TPS cytological criteria and fluorescence parameters on voided urine slides after LBC procedure. This algorithm would allow automatic and rapid screening with both FMi and UCCy. This rapid shift to automatic dual screening can explain the short series of patients in this preliminary retrospective study. The selection bias was reduced by the homogeneity of our study: strict criteria of inclusion and exclusion, precise definition of the two different fluorescent signals, normal and tumoral-type, intervention of a single experienced pathologist and a single university urologist surgeon that limited inter observer variations for FMi and cystoscopy.

\section{Conclusions}

The association of UCCy with FMi effectively addresses the issue of LGUN detection. According to our results, with the local cytopathology expertise, FMi performed on urothelial cells from voided urine is more sensitive than UCCy, making the former a valuable complementary method in the diagnosis of urothelial tumors and follow-up of patients.

\section{Acknowledgments}

None to declare.

\section{Financial Disclosure}

None to declare.

\section{Conflict of Interest}

This method of detecting tumor cells by fluorescence signals is protected by the United States Patent US8597905 B2 2013.2. 
The owners are Fabre M, Ferlicot S, Fontaine Aupart MP, Steenkeste K, Eschwege P.

\section{Informed Consent}

In this retrospective study, informed consent was not applicable.

\section{Authors Contributions}

Drak Alsibai K: formal analysis, investigation, methodology, visualization, writing original draft. Daste G: formal analysis, investigation, visualization, writing original draft, writing review and editing. Ferlicot S: formal analysis, data curation, investigation, conceptualization. Fabre M: data curation, methodology, writing original draft, writing review and editing. Salleron J: data curation, formal analysis, investigation, methodology. Steenkeste K: data curation, formal analysis, investigation, validation. Hammoudi Y: data curation, investigation, validation, resources. Fontaine-Aupart MP: data curation, project administration, conceptualization, supervision. Eschwege P: data curation, project administration, conceptualization, supervision.

\section{Data Availability}

The authors declare that data supporting the findings of this study are available within the article.

\section{References}

1. Bray F, Ferlay J, Soerjomataram I, Siegel RL, Torre LA, Jemal A. Global cancer statistics 2018: GLOBOCAN estimates of incidence and mortality worldwide for 36 cancers in 185 countries. CA Cancer J Clin. 2018;68(6):394424.

2. Shen YJ, Zhu YP, Ye DW, Yao XD, Zhang SL, Dai B, Zhang HL, et al. Narrow-band imaging flexible cystoscopy in the detection of primary non-muscle invasive bladder cancer: a "second look" matters? Int Urol Nephrol. 2012;44(2):451-457.

3. Grimbergen MC, van Swol CF, Jonges TG, Boon TA, van Moorselaar RJ. Reduced specificity of 5-ALA induced fluorescence in photodynamic diagnosis of transitional cell carcinoma after previous intravesical therapy. Eur Urol. 2003;44(1):51-56.

4. Wiener HG, Mian C, Haitel A, Pycha A, Schatzl G, Marberger M. Can urine bound diagnostic tests replace cystoscopy in the management of bladder cancer? J Urol. 1998;159(6):1876-1880.

5. Fontaniere B, Ranchere-Vince D, Landry JL, Colombel M, Chopin D, Gattegno B. [Quality criteria in urinary cytology for tumor diagnosis]. Prog Urol. 2001;11(5):867875.

6. Glatz K, Willi N, Glatz D, Barascud A, Grilli B, Herzog
M, Dalquen P, et al. An international telecytologic quiz on urinary cytology reveals educational deficits and absence of a commonly used classification system. Am J Clin Pathol. 2006;126(2):294-301.

7. Strittmatter F, Buchner A, Karl A, Sommer ML, Straub J, Tilki D, Hennenberg M, et al. Individual learning curve reduces the clinical value of urinary cytology. Clin Genitourin Cancer. 2011;9(1):22-26.

8. Muus Ubago J, Mehta V, Wojcik EM, Barkan GA. Evaluation of atypical urine cytology progression to malignancy. Cancer Cytopathol. 2013;121(7):387-391.

9. Piaton E, Advenier AS, Benaim G, Petrucci MD, Lechevallier FM, Ruffion A. [Atypical urothelial cells (AUC): A Bethesda-derived wording applicable to urinary cytopathology]. Ann Pathol. 2011;31(1):11-17.

10. Rosenthal DL, Wojcik EM, Kurtycz DFI. Paris system for reporting urinary cytopathology. New York; Springer. 2016. p. 159.

11. Hassan M, Solanki S, Kassouf W, Kanber Y, Caglar D, Auger M, Brimo F. Impact of implementing the paris system for reporting urine cytology in the performance of urine cytology: a correlative study of 124 cases. Am J Clin Pathol. 2016;146(3):384-390.

12. Miki Y, Neat M, Chandra A. Application of The Paris System to atypical urine cytology samples: correlation with histology and UroVysion((R)) FISH. Cytopathology. 2017;28(2):88-95.

13. Roy M, Kaushal S, Jain D, Seth A, Iyer VK, Mathur SR. An institutional experience with The Paris System: A paradigm shift from ambiguous terminology to more objective criteria for reporting urine cytology. Cytopathology. 2017;28(6):509-515.

14. Suh J, Go H, Sung C, Baek S, Hwang H, Jeong S, Cho Y. Modification of The Paris System for urinary tract washing specimens using diagnostic cytological features. Cytopathology. 2017;28(6):516-523.

15. Glass R, Rosen L, Chau K, Sheikh-Fayyaz S, Farmer P, Coutsouvelis C, Slim F, et al. Analysis of the cytomorphological features in atypical urine specimens following application of the Paris system for reporting urinary cytology. Acta Cytol. 2018;62(1):54-61.

16. Proctor I, Stoeber K, Williams GH. Biomarkers in bladder cancer. Histopathology. 2010;57(1):1-13.

17. Budman LI, Kassouf W, Steinberg JR. Biomarkers for detection and surveillance of bladder cancer. Can Urol Assoc J. 2008;2(3):212-221.

18. Mian C, Maier K, Comploj E, Lodde M, Berner L, Lusuardi L, Palermo S, et al. uCyt+/ImmunoCyt in the detection of recurrent urothelial carcinoma: an update on 1991 analyses. Cancer. 2006;108(1):60-65.

19. Daniely M, Rona R, Kaplan T, Olsfanger S, Elboim L, Freiberger A, Lew S, et al. Combined morphologic and fluorescence in situ hybridization analysis of voided urine samples for the detection and follow-up of bladder cancer in patients with benign urine cytology. Cancer. 2007;111(6):517-524.

20. Bubendorf L, Piaton E. UroVysion(R) multiprobe FISH in the triage of equivocal urinary cytology cases. Ann Pathol. 2012;32(6):e52-e56. 
21. Mahnert B, Tauber S, Kriegmair M, Schmitt UM, Hasholzner U, Reiter W, Hofmann K, et al. BTA-TRAK-a useful diagnostic tool in urinary bladder cancer? Anticancer Res. 1999;19(4A):2615-2619.

22. Ramakumar S, Bhuiyan J, Besse JA, Roberts SG, Wollan PC, Blute ML, O'Kane DJ. Comparison of screening methods in the detection of bladder cancer. J Urol. 1999;161(2):388-394.

23. Leyh H, Marberger M, Conort P, Sternberg C, Pansadoro V, Pagano F, Bassi P, et al. Comparison of the BTA stat test with voided urine cytology and bladder wash cytology in the diagnosis and monitoring of bladder cancer. Eur Urol. 1999;35(1):52-56.

24. Hwang EC, Choi HS, Jung SI, Kwon DD, Park K, Ryu SB. Use of the NMP22 BladderChek test in the diagnosis and follow-up of urothelial cancer: a cross-sectional study. Urology. 2011;77(1):154-159.

25. Oeyen E, Hoekx L, De Wachter S, Baldewijns M, Ameye F, Mertens I. Bladder cancer diagnosis and follow-up: the current status and possible role of extracellular vesicles. Int J Mol Sci. 2019;20(4):821-839.

26. Kassouf W, Traboulsi SL, Schmitz-Drager B, Palou J, Witjes JA, van Rhijn BW, Grossman HB, et al. Followup in non-muscle-invasive bladder cancer-International Bladder Cancer Network recommendations. Urol Oncol. 2016;34(10):460-468.

27. Millholland JM, Li S, Fernandez CA, Shuber AP. Detection of low frequency FGFR3 mutations in the urine of bladder cancer patients using next-generation deep sequencing. Res Rep Urol. 2012;4:33-40.

28. Witjes JA, Morote J, Cornel EB, Gakis G, van Valenberg FJP, Lozano F, Sternberg IA, et al. Performance of the bladder EpiCheck methylation test for patients under surveillance for non-muscle-invasive bladder cancer: results of a multicenter, prospective, blinded clinical trial. Eur Urol Oncol. 2018;1(4):307-313.

29. Kavalieris L, O'Sullivan PJ, Suttie JM, Pownall BK, Gilling PJ, Chemasle C, Darling DG. A segregation index combining phenotypic (clinical characteristics) and genotypic (gene expression) biomarkers from a urine sample to triage out patients presenting with hematuria who have a low probability of urothelial carcinoma. BMC Urol. 2015; 15:23.

30. Lotan Y, O'Sullivan P, Raman JD, Shariat SF, Kavalieris L, Frampton C, Guilford P, et al. Clinical comparison of noninvasive urine tests for ruling out recurrent urothelial carcinoma. Urol Oncol. 2017;35(8):531 e515-531 e522.

31. Chang SS, Boorjian SA, Chou R, Clark PE, Daneshmand S, Konety BR, Pruthi R, et al. Diagnosis and treatment of non-muscle invasive bladder cancer: AUA/SUO guideline. J Urol. 2016;196(4):1021-1029.

32. Follow up of patients with NMIBC: EAU Guidelines: https://uroweb.org/guideline/non-muscle-invasive-bladder-cancer/\#8.

33. Piaton E, Faynel J, Hutin K, Ranchin MC, Cottier M. Conventional liquid-based techniques versus Cytyc
Thinprep processing of urinary samples: a qualitative approach. BMC Clin Pathol. 2005;5:9.

34. Kim JY, Kim HJ. A Comparison Between ThinPrep Monolayer and Cytospin cytology for the detection of bladder cancer. Korean J Urol. 2014;55(6):390-394.

35. Millot C, Bondza-Kibangou P, Millot JM, Lallemand A, Manfait M. Autofluorescence spectroscopy of malpighian epithelial cells, as a new tool for analysis of cervical cancer precursors. Histol Histopathol. 2003;18(2):479-485.

36. Tauber S, Schneede P, Liedl B, Liesmann F, Zaak D, Hofstetter A. Fluorescence cytology of the urinary bladder. Urology. 2003;61(5):1067-1071.

37. Steenkeste K, Lecart S, Deniset A, Pernot P, Eschwege P, Ferlicot S, Leveque-Fort S, et al. Ex vivo fluorescence imaging of normal and malignant urothelial cells to enhance early diagnosis. Photochem Photobiol. 2007;83(5):11571166.

38. Olivo M, Lau W, Manivasager V, Bhuvaneswari R, Wei $\mathrm{Z}$, Soo KC, Cheng $\mathrm{C}$, et al. Novel photodynamic diagnosis of bladder cancer: ex vivo fluorescence cytology using hypericin. Int J Oncol. 2003;23(6):1501-1504.

39. Moskowitz CS, Pepe MS. Comparing the predictive values of diagnostic tests: sample size and analysis for paired study designs. Clin Trials. 2006;3(3):272-279.

40. Renshaw AA. Subclassifying atypical urinary cytology specimens. Cancer. 2000;90(4):222-229.

41. Brimo F, Vollmer RT, Case B, Aprikian A, Kassouf W, Auger M. Accuracy of urine cytology and the significance of an atypical category. Am J Clin Pathol. 2009;132(5):785793.

42. Rosenthal DL, Vandenbussche CJ, Burroughs FH, Sathiyamoorthy S, Guan H, Owens C. The Johns Hopkins Hospital template for urologic cytology samples: part I-creating the template. Cancer Cytopathol. 2013;121(1):15-20.

43. Granados R, Duarte JA, Corrales T, Camarmo E, Bajo P. Applying the Paris system for reporting urine cytology increases the rate of atypical urothelial cells in benign cases: a need for patient management recommendations. Acta Cytol. 2017;61(1):71-76.

44. Savic S, Zlobec I, Thalmann GN, Engeler D, Schmauss M, Lehmann K, Mattarelli G, et al. The prognostic value of cytology and fluorescence in situ hybridization in the follow-up of nonmuscle-invasive bladder cancer after intravesical Bacillus Calmette-Guerin therapy. Int J Cancer. 2009;124(12):2899-2904.

45. Tilki D, Burger M, Dalbagni G, Grossman HB, Hakenberg OW, Palou J, Reich O, et al. Urine markers for detection and surveillance of non-muscle-invasive bladder cancer. Eur Urol. 2011;60(3):484-492.

46. Yang F, Zhou Q, Meng L, Xing N. IMP3 is a biomarker for non-muscle-invasive urothelial carcinoma of the bladder associated with an aggressive phenotype. Medicine. 2019;98(27):1-7.

47. Fabre M, Ferlicot S, Fontaine-Aupart MP, Steenkeste K, Eschwege P. Method of detecting tumor cells by fluorescence signals. United State Patent US20120264165A1. 